\title{
Erratum to: Developmental and individual differences in Chinese writing
}

\author{
Connie Qun Guan • Feifei Ye • Richard K. Wagner • \\ Wanjin Meng
}

\section{Erratum to: Read Writ \\ DOI 10.1007/s11145-012-9405-4}

The following information was missed out in the original published article and the following erratum is published at the author's request:

Acknowledgments This research was supported by Engineering Research Institute Foundations of USTB Grant (YJ2012-019) entitled Research on Invariance Model of Writing to Connie Qun Guan, sponsored by National Institute of Education Science (NIES) Grant (Number GY2012013) awarded to Connie Qun Guan and Wanjin Meng, and funded by NICHD Grant P50HD052120 to Richard K. Wagner.

The online version of the original article can be found under doi:10.1007/s11145-012-9405-4.

C. Q. Guan

University of Science and Technology Beijing, Beijing, China

C. Q. Guan

Florida Center for Reading Research, Florida State University, West Call Street,

Tallahassee, FL 32306, USA

F. Ye

University of Pittsburgh, Pittsburgh, PA, USA

R. K. Wagner ( $₫)$

Department of Psychology, Florida State University, 1107 West Call Street,

P.O. Box 3034301, Tallahassee, FL 32306-4301, USA

e-mail: rkwagner@psy.fsu.edu

W. Meng $(\square)$

National Institute of Education Sciences, Beijing, China

e-mail: wanjinmeng@yahoo.com 